\title{
Nonlinearity of a Voltage-Gated Potassium Channel Revealed by the Mechanical Susceptibility
}

\author{
Amila Ariyaratne and Giovanni Zocchi* \\ Department of Physics and Astronomy, University of California Los Angeles, Los Angeles, California 90095-1547, USA
} (Received 13 March 2012; revised manuscript received 15 October 2012; published 11 February 2013)

\begin{abstract}
The voltage-gated potassium channel from Aeropyrum pernix operates by coupling the voltage-driven motion of a charged group of amino acids to the opening and closing of the pore. In this experiment, we drive this charged group with an ac field and observe the effect on the gating. The measurements for different frequencies and amplitudes of the forcing reveal an essential nonlinearity in the mechanical behavior of the molecule. Within a continuum-mechanics description, we extract the effective dissipation parameter $\gamma$ for this conformational motion and find $\gamma \approx 0.2 \mathrm{~g} / \mathrm{s}$, similar to recent nanorheology measurements on the conformational motion of an enzyme.
\end{abstract}

DOI: 10.1103/PhysRevX.3.011010

\section{INTRODUCTION}

Voltage-gated ion channels are membrane proteins that allow the conduction of a specific ion across the cell membrane (with few exceptions where the channel allows more than one type of ion permeation) by opening and closing a pore in response to changes in the electrostaticpotential difference across the membrane. The family of voltage-gated potassium channels, in particular, has been studied extensively. The standard electrophysiology measurement consists of applying a dc pulse to (i.e., polarizing) the membrane and observing the current in time using a voltage-clamp configuration [1]. By contrast, not much work is reported on the ac response of the ion channel, by which we mean the response to an oscillating voltage imposed across the membrane. If the channel were a linear device, one could argue that the response to a step function (the dc response) contains all the information, but the response to a step function does not contain all the information for a nonlinear system, in which case ac measurements may unravel new properties. We are aware of only a few ac measurements on channels, and that too on channels that are simple pores (i.e., not voltage gated). Ervin et al. [2] performed measurements on the alpha-hemolysin ion channel using dc-biased ac signals. By superimposing an ac signal onto a dc membrane voltage, they studied the blocking events of the channel due to the interaction with other proteins and DNA. However, the alpha-hemolysin channel is not a voltage-gated ion channel but acts like a pore in the membrane at the electrophysiological voltages used. Misakian et al. [3] studied the frequency response of the alpha-hemolysin ion channel and concluded that in the frequency range $30-120 \mathrm{~Hz}$ the channel behaves like a

\footnotetext{
*zocchi@physics.ucla.edu
}

Published by the American Physical Society under the terms of the Creative Commons Attribution 3.0 License. Further distribution of this work must maintain attribution to the author(s) and the published article's title, journal citation, and DOI.
Subject Areas: Biological Physics, Interdisciplinary Physics, Nonlinear Dynamics

resistor. Our present work differs from the above because, unlike the alpha-hemolysin channel that remains open independent of the membrane voltage, the voltage-gated potassium channel opens and closes in response to the membrane voltage. Our ac measurements unravel a fundamental nonlinearity of this channel at physiological applied voltages that was not known before.

Ion channels in the environment of the nerve cell are always subjected to ac signals. Therefore, understanding the response to ac signals should be important for the understanding of signal transmission in the neuron. We come to this problem, however, from yet another perspective, which is to investigate the rheological properties of the folded state of proteins (the rheology of the channel in this case), as will become clear in the following.

The major difficulty in studying the ac response of ion channels is the capacitive current at the lipid bilayer. As the bilayer acts as a capacitor, an oscillating voltage applied across the membrane gives rise to an oscillating capacitive current with an amplitude proportional to the applied voltage and frequency. The single-channel current is easily overwhelmed by this capacitive current in an ac measurement. In our study, we subtract the capacitive current by applying the same biasing voltage to the bilayer and to a separate tunable capacitor; we find that our setup is suitable for measuring multichannel activity up to frequencies of approximately $100 \mathrm{~Hz}$.

The ion channel of this study is the voltage-gated potassium channel from Aeropyrum pernix (KvAP). Ruta and coworkers characterized the KvAP channel functionally [4] and found that it has a conductivity of approximately $170 \mathrm{pS}$; the channel undergoes inactivation when subjected to a prolonged depolarizing voltage similar to other $\mathrm{Kv}$ channels, and pore-blocking toxins bind to block KvAP in a similar manner to other $\mathrm{Kv}$ channels. The structure of KvAP was solved in 2003 by the MacKinnon lab [5] (see Fig. 1). It is a tetramer, each unit comprising a voltage-sensing domain (VSD) and a pore-forming domain. The central pore domain is the ion-conduction pathway. The S4 helix of the VSD contains five arginine amino-acid groups 


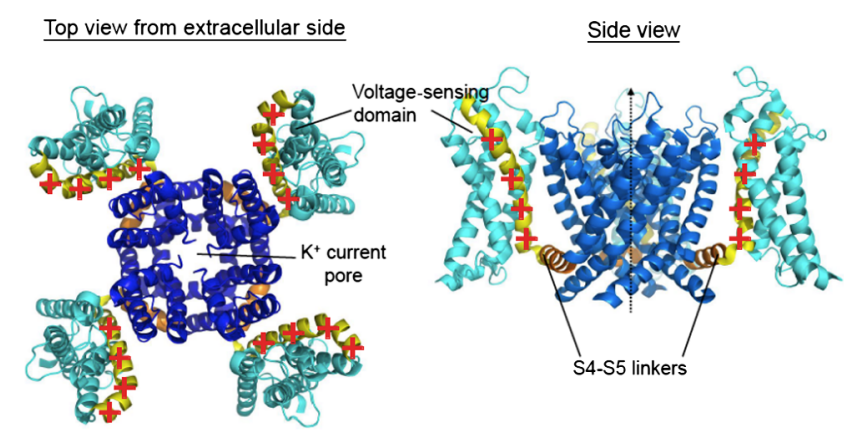

FIG. 1. Structure of a $\mathrm{Kv}$ ion channel (Kv1.2-2.1) with a similar structure to that of KvAP. The functional channel is a tetramer with the central pore domain (dark blue) forming the ion-conduction pathway. Red crosses indicate the positively charged arginine amino acids involved in gating.

that are positively charged; four of these groups contribute to voltage-dependent gating [4]. It is well established that these arginines move in response to the electric field across the membrane. This motion has in fact been measured in several ways: by detecting the corresponding current (gating current) $[6,7]$ and more recently by Förster resonance energy transfer $[8,9]$, and it gives a displacement of approximately $2 \AA$. A study using voltage-clamp fluorometry [10] found a displacement of approximately 6-8 $\AA$, while biotin-avidin accessibility indicates even larger movements of approximately 15-20 ̊ [11]. In our study, we use an averaged value for the displacement of the arginines based on these different experimental results to calculate an effective spring constant for the gating charge movement discussed in the paper. The motion of the VSDs is coupled to the pore that opens and closes as the VSDs move in the bilayer.

The behavior of a single ion channel is stochastic. When a depolarizing voltage is applied across the membrane, the single-channel current is a telegraph signal with the probability that the channel is open, given by the graph of Fig. 2 . The meaning of this curve is as follows. At the resting potential (approximately $-60 \mathrm{mV}$ ), the channels are closed. If, starting from this state, the voltage is stepped up to the value $V$, after an approximately 50-ms transient, the channels flicker open and closed with an open probability $p(V)$, shown in Fig. 2.

However, this state is not exactly a steady state, due to a slow inactivation process by which a channel can jump from the open to a different closed state that is termed inactive because it remains closed unless the channel is recycled through the resting potential for a sufficient time [12]. This inactivation of ion channels is shown in Fig. 3, which presents a typical current trace from a multichannel recording. The channels are hyperpolarized at $-120 \mathrm{mV}$ prior to a depolarizing potential step to $60 \mathrm{mV}$ at $t=0$. The fast rise of the current is due to the stochastic process of the channels going from the closed to the open state. The current reaches a maximum value and then decreases slowly with a time constant of $0.4-1 \mathrm{~s}$. This decrease is due

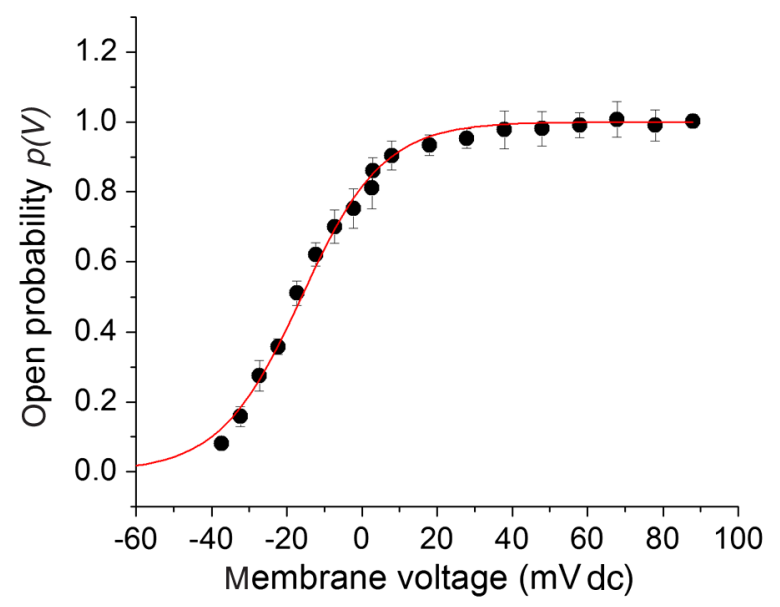

FIG. 2. Dependence of the open probability (the fraction of time the channel is open) on the membrane voltage. The open probability at voltage $V$ is measured from the peak current $I_{0}$ obtained by depolarizing the channels with a voltage step ending at $V$. The conductance $I_{0} / V$, plotted vs $V$ and normalized to 1 at high $V$, is shown in the figure. The solid line is produced by a fit using Eq. (3). Here, as in the rest of the paper, the ion channel is the KvAP channel with the internal cysteine removed (see Section II), and the symmetric salt conditions of $150 \mathrm{mM} \mathrm{KCl}$ are used. The data in the graph are from the same bilayer. The channels are closed with probability 1 below approximately $-40 \mathrm{mV}$ and open with probability 1 above approximately $+20 \mathrm{mV}$. In the neuron, the typical resting potential is $-70 \mathrm{mV}$ and the spike of the action potential is approximately $50 \mathrm{mV}$.

to the inactivation process characteristic of voltagedependent ion channels.

Our experiment follows in the tracks of many years of studies by the Bezanilla group [13] and others [14-16], which established that the motion of charged groups within

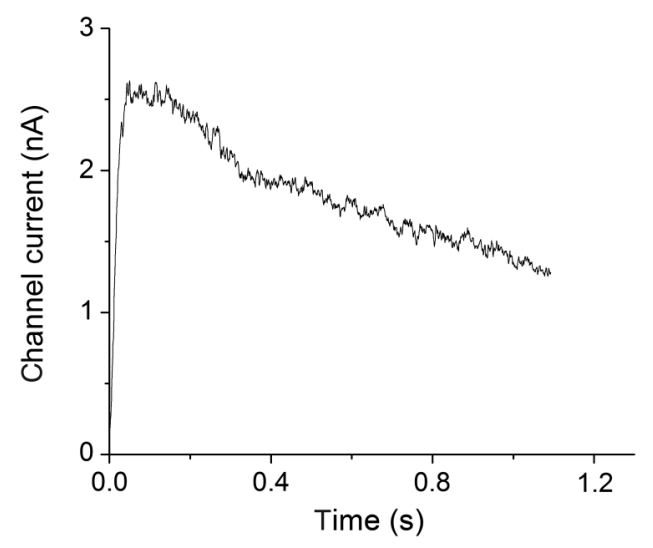

FIG. 3. A typical multichannel current trace. The depolarizing step potential was $60 \mathrm{mV}$; i.e., starting from a holding potential of $-120 \mathrm{mV}$ where channels are closed, the membrane potential is brought to $+60 \mathrm{mV}$ in one step at $t=0$. The current shows a "fast" rise (an approximately 50-ms time scale) corresponding to the stochastic opening of the channels, a plateau, and a "slow" exponential decay caused by the "inactivation" process. There are roughly 250 active channels in this bilayer. 


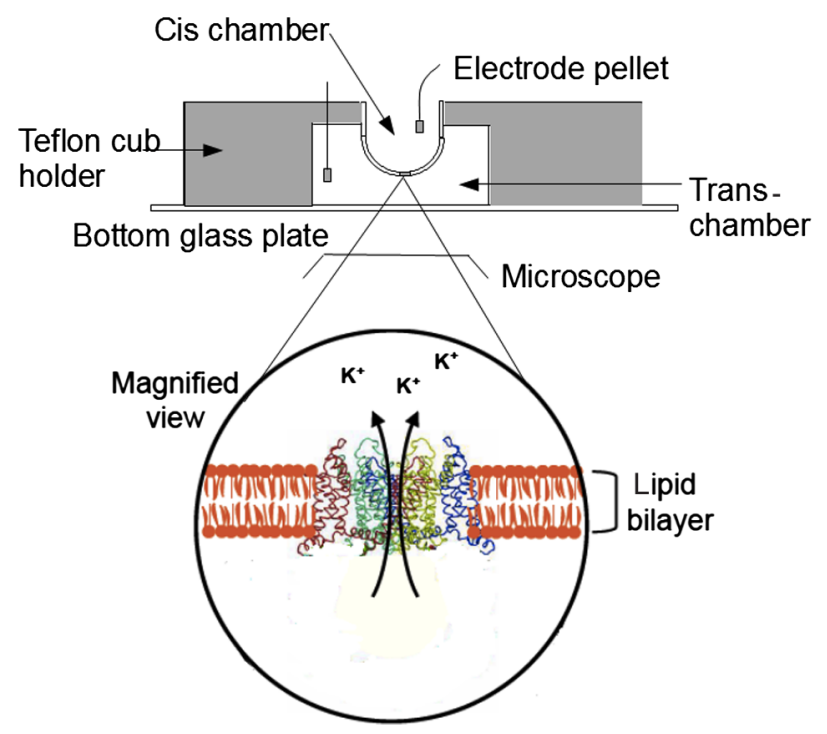

FIG. 4. The planar-lipid-bilayer system. The artificial bilayer is stretched across an approximately $100-\mu \mathrm{m}$ hole crafted in a plastic test tube mounted on a Teflon holder. Electrical connections to the bilayer chamber are made via $\mathrm{Ag} / \mathrm{AgCl}$ electrodes. The whole system is mounted onto the $x-y$ stage of an inverted microscope.

the VSD is responsible for the gating mechanism. This motion is driven by the electric field generated by the potential difference across the membrane. Therefore, applying an ac membrane potential to the setup of Fig. 4 can be thought of, to some extent, as a nanorheology experiment similar to [17]: An oscillating force is applied to the voltage-sensing domain, driving the motion of parts of the domain (in particular, the S4 helix containing the arginines) relative to the rest of the structure and the membrane. The difference with our recent nanorheology experiment on the folded protein [17-19] is that we do not directly measure the amplitude of the motion of the voltage-sensing domain but instead measure the current. Even then, the results can be interpreted in a simple way that reveals the mechanical properties of the channel. In summary, we measure the current resulting from the ac excitation for different frequencies in the range of $5-150 \mathrm{~Hz}$ and different amplitudes of the applied sinusoidal voltage in the range of $10-100 \mathrm{mV}$. The data show that the behavior of the channel is fundamentally nonlinear in this range. Here, "fundamentally nonlinear" refers to a form of nonlinearity that is beyond the well-known nonlinearity depicted by the response curve in Fig. 2. All the measurements can be summarized in terms of one simple mechanical model that we use to suggest a form for this nonlinearity in terms of the materials properties associated with the motion of the VSD.

\section{MATERIALS AND METHODS}

The ion channel used in this study is the KvAP, a voltagegated potassium channel. We express the channel from a gene slightly modified (by site-directed mutagenesis) from the cloned wild-type KvAP gene, in that the native cysteine at site 247 is replaced with a serine. This modification, which is introduced for the sake of a different project, does not alter the activity of the channel.

\section{A. Expression and purification of the KvAP protein}

We use the expression vector pQE60 to express the protein in Escherichia coli cells (Stratagene XL1-Blue). The transformation of the plasmid vector into the cells is achieved by giving a heat pulse of $42{ }^{\circ} \mathrm{C}$ for a duration of $45 \mathrm{~s}$. All of the procedures below are performed at room temperature and are adapted from [4]. The protein expressed in $10 \mathrm{~g}$ of $E$. coli is resuspended in $50 \mathrm{~mL}$ of lysis buffer (50-mM Tris at $\mathrm{pH} 8.0,100-\mathrm{mM} \mathrm{KCl}, 0.2-\mathrm{mg} / \mathrm{mL}$ lysozyme, $2-\mu \mathrm{g} / \mathrm{mL}$ DNase, 2-mM beta-mercaptoethanol and protease inhibitor cocktail) and lysed using a French press. Decylmaltoside detergent at $40 \mathrm{mM}$ (DM, Anatrace) is used for $3 \mathrm{~h}$ to extract the channel protein from the lysate. The supernatant obtained by centrifuging the lysate-DM mixture is passed through a Talon affinity column (Clontech) so that the his-tagged ion channel binds to the beads. Before adding the protein supernatant, the Talon beads are washed with $30 \mathrm{~mL}$ of wash buffer (20-mM Tris at $\mathrm{pH} 8.0,100-\mathrm{mM} \mathrm{KCl}$, $10-\mathrm{mM}$ imidazole, and 5-mM DM). Then, the beadsupernatant mixture is gently rotated for $1 \mathrm{~h}$ and is subsequently passed through the column 4 times to retain all the beads in the column. A wash buffer $(30 \mathrm{~mL})$ is used to remove nonspecifically bound species from the beads followed by $10 \mathrm{~mL}$ of elution buffer (similar to the wash buffer but with $400-\mathrm{mM}$ imidazole) to elute the ion channel. 1.5 units of thrombin (Sigma) are added to each milligram of channel protein to cleave the his tag overnight. The protein treated with thrombin is run in a size-exclusion Superdex200 column (GE Healthcare) in a high-performance liquidchromatography buffer (20-mM Tris at $\mathrm{pH} 7.5,100-\mathrm{mM}$ $\mathrm{KCl}$, and 5-mM DM) and concentrated to $10 \mathrm{mg} / \mathrm{mL}$.

\section{B. Reconstitution of the KvAP ion channel into lipid vesicles}

The lipid 1,2-diphytanoyl-sn-glycero-3-phosphocholine (DPhPC) (Avanti) is used to make vesicles; the procedure below is adapted from [20]. First, the lipid in chloroform is dried using a pure nitrogen flow, followed by adding approximately $250 \mu \mathrm{L}$ pentene and drying. The dried lipid is then put under a vacuum for $1 \mathrm{~h}$ before dissolving in a reconstitution buffer [10-mM 4-(2-hydroxyethyl)-1piperazineethanesulfonic acid (HEPES) $\mathrm{KOH}$ at $\mathrm{pH} 7.4$ and $450-\mathrm{mM} \mathrm{KCl}$ ] for a lipid concentration of $20 \mathrm{mg} / \mathrm{mL}$ obtained by rapid vortexing for $30 \mathrm{~min}$. The resulting lipid solution is then sonicated for 20-30 min to form unilamellar vesicles and is dissolved in $10-\mathrm{mM}$ DM for $30 \mathrm{~min}$. The concentrated ion-channel protein is then added to the lipid vesicle solution, to a lipid-to-protein ratio of $1(\mathrm{wt} / \mathrm{wt})$. The detergent concentration is then raised to $17.5 \mathrm{mM}$, and the mixture is then incubated for $2 \mathrm{~h}$ with gentle vortexing every $20 \mathrm{~min}$. The next phase of reconstitution is 
to remove the DM. First, the vesicle-protein solution is passed through three spin-desalting columns (Pierce). Then, an adequate amount of detergent-absorbing biobeads (Bio-Rad) that are washed prior to use (first in methanol, then in deionized water, and finally in reconstitution buffer) is put to the vesicle-protein solution and is kept at $4{ }^{\circ} \mathrm{C}$. The solution is then put in new biobeads every $12 \mathrm{~h}$ for another 3 times. At the end of the fourth cycle, the detergent-free proteoliposomes are flash frozen (using an ethanol-dry-ice bath) in small aliquots and are stored at $-80^{\circ} \mathrm{C}$ for later use.

\section{Electrophysiology setup}

A planar-lipid-bilayer setup is used for measuring the activity of the ion channels. The melt-and-shave method described in Wonderlin et al. [21] is used to make a coneshaped dent in a plastic Ultra-Clear (Beckman Coulter) tube. Then, the dent is shaved with a sharp blade to obtain an approximately $150-\mu \mathrm{m}$ circular aperture and is fixed to a Teflon holder that serves as the extracellular chamber (transchamber). The whole system is then mounted on an inverted microscope so that the bilayer formation could be viewed (Fig. 4). The DPhPC lipid is taken fresh from the $-80^{\circ} \mathrm{C}$ freezer for each experimental run and is dried according to the method mentioned above. n-Decane is added to the dried lipid to a lipid concentration of $20 \mathrm{mg} / \mathrm{ml}$. Then, a small drop of lipid $(<0.5 \mu \mathrm{L})$ is put onto the aperture and is allowed to dry. The two chambers are then filled with a buffer containing 150-mM KCl, 10-mM HEPES-KOH at $\mathrm{pH} \mathrm{7.4,} \mathrm{and} \mathrm{a} \mathrm{second} \mathrm{small} \mathrm{drop} \mathrm{of} \mathrm{lipid} \mathrm{is} \mathrm{added.} \mathrm{The}$ removal of excess lipid from the aperture using a small glass spatula results in the formation of a bilayer. Once a stable bilayer is available, a dose of channel vesicles is added to the cis chamber from a fresh aliquot of channel vesicles (thawed and sonicated for approximately $10 \mathrm{~s}$ ). The vesicle fusion to the bilayer could be seen as spiking events when the holding voltage is kept at $-120 \mathrm{mV}$ and the process is accelerated by stirring the cis chamber with a glass rod.

Experiments at different frequencies and amplitudes of the imposed sinusoidal membrane potential are performed. Ion-channel currents are detected using a home-built voltage-clamp amplifier with a feedback resistor of $22 \mathrm{M} \Omega$. The capacitive current through the bilayer is subtracted by using a mirror voltage-clamp amplifier connected to a tunable capacitor (Fig. 5). The output from the voltage-clamp amplifier and the mirror voltage-clamp amplifier is fed into a low-noise preamplifier (SR560, Stanford Research Systems) in the A-B mode. The signal is digitized using an analog-to-digital converter (NI USB6009 , National Instruments) and recorded using the software LabView Signal Express (National Instruments). A battery-powered pulse-generating circuit is used to generate the voltage protocol across the bilayer (Fig. 6).

The method employed to subtract capacitive currents assumes that the measured current is the sum of the channels' current and the bilayer's capacitive current.

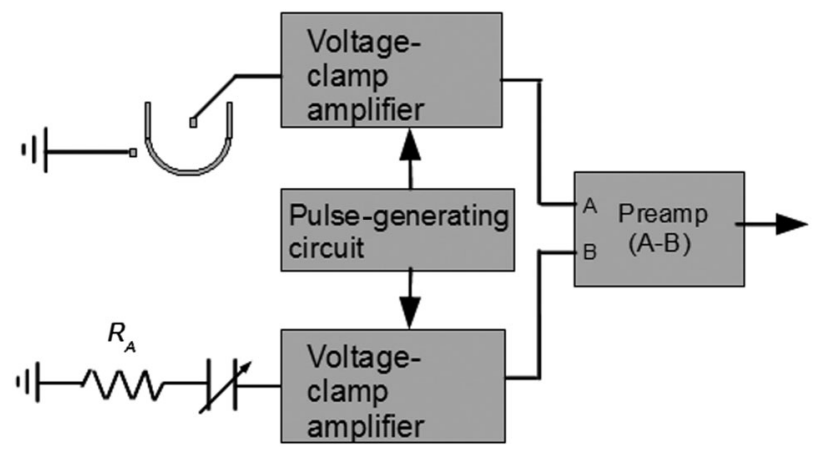

FIG. 5. Diagram of the electronics for the electrophysiology setup. The voltage-clamp amplifiers were home built with identical components. The variable capacitor circuit has the purpose of canceling the bilayer's capacitive current by subtracting a similar capacitive current from the signal. $R_{A}=10 \mathrm{~K} \Omega$, and the tunable capacitor has a range of $20-150 \mathrm{pF}$.

Conceptually, the method consists in measuring the effective capacitance of the bilayer; setting a dummy capacitive circuit to that value of the capacitance; and then employing, in the experiments, the same excitation and readout for the real system and the dummy electronic circuit. The final signal is the difference of the two signals. Operationally, for each measurement, this capacitive-current subtraction is achieved as follows. First, a stable bilayer is obtained with about $100+$ ion channels on it. A bilayer with $100+$ channels is verified with dc pulses. Once a stable bilayer with ion channels is obtained, an ac pulse at $60 \mathrm{mV} \mathrm{rms} \mathrm{is}$ applied. The frequency is around $100-150 \mathrm{~Hz}$. When this signal is maintained for about $1 \mathrm{~min}$, all the ion channels become inactive and the current that is measured is purely the capacitive current. A second head-stage amplifier connected to a tunable capacitor circuit is now used to match the current produced by the head-stage amplifier connected to the bilayer assembly (in practice, we take the difference of the two signals and tune the capacitor for zero current; see Figs. 5 and 7). Once an optimum position for the tunable capacitor is found where the currents produced by both head-stage amplifiers match, the capacitive compensation is kept fixed over the course of a measurement.

In the measurements, the difference of the output from the two head-stage amplifiers is taken, so the capacitive portion of the current is (largely) eliminated.

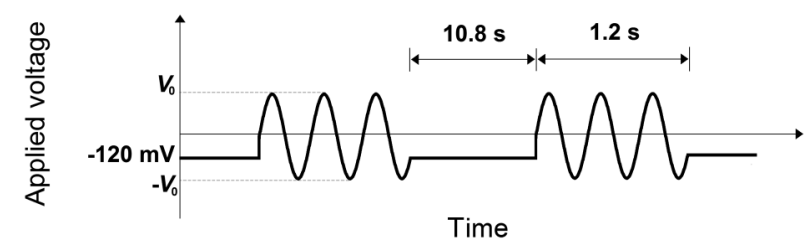

FIG. 6. Pulse protocol. A resting potential of $-120 \mathrm{mV}$ is maintained for $10.8 \mathrm{~s}$ in order for the channels to recover fully from inactivation. The resting potential of $-120 \mathrm{mV}$ is followed by a sinusoidal signal centered around $0 \mathrm{mV}$ lasting for $1.2 \mathrm{~s}$. The control parameters of the experiment are the amplitude and the frequency of the sinusoidal signal. 


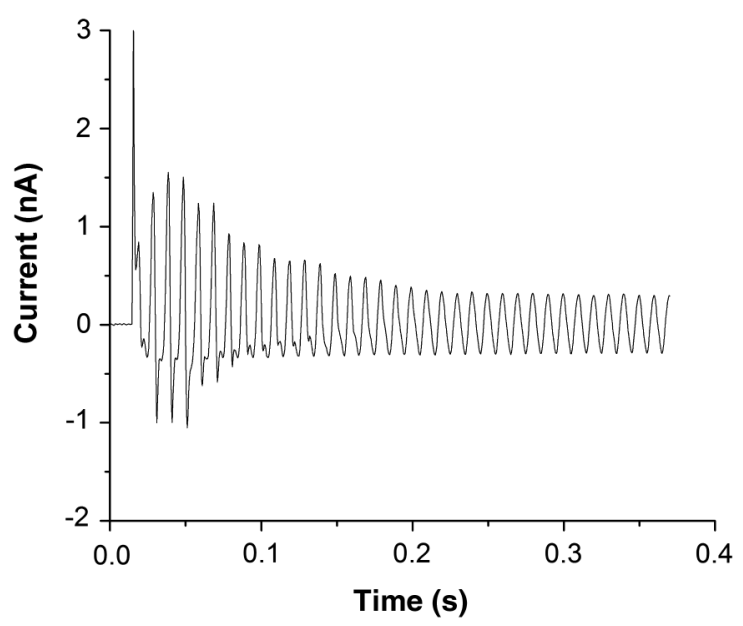

(a)

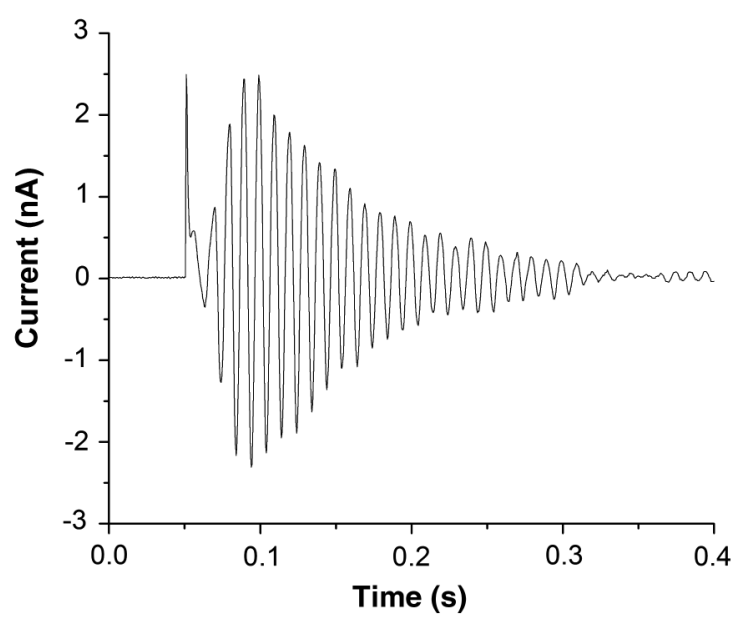

(b)

FIG. 7. The capacitive compensation demonstrated for a 100$\mathrm{Hz}$ signal. (a) Current trace obtained with $100 \mathrm{~Hz}$ driving without substracting the capacitive current. At long times, the oscillation amplitude tends to a constant value that reflects the capacitive current only. (b) Current trace (from a different sample) obtained with capacitive-current substraction. At long times, the current goes to zero.

Using this approach, it is possible to effectively cancel the background capacitive current up to a signalto-background ratio of about 20-50 in the frequency range from 5 to $175 \mathrm{~Hz}$ for multichannel recordings with approximately 100 channels (Fig. 7). All recordings are obtained for symmetric salt conditions in the cis and transchambers: $150-\mathrm{mM} \mathrm{KCl,} \mathrm{10-mM} \mathrm{HEPES} \mathrm{at} \mathrm{pH} \mathrm{7.4.}$

\section{Data analysis}

The peak value of current from a trace is obtained by averaging 10 sample points about the maximum value of the peak (acquisition frequency of $4 \mathrm{kHz}$ ). A data point reported in a plot such as Fig. 8 is obtained by averaging 5-6 peak-current values from successive voltage protocols

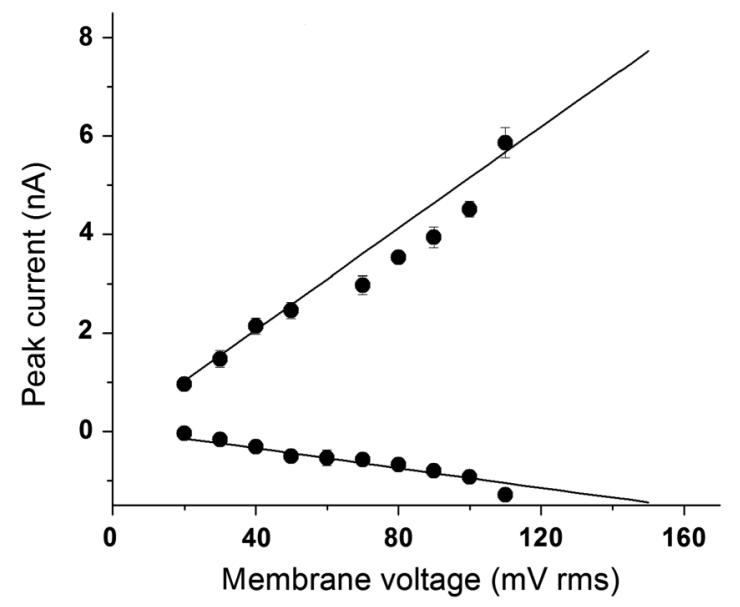

FIG. 8. The peak current (for both positive and negative swings) measured for the varying amplitude of the driving voltage $V_{0}$ for $\nu=5 \mathrm{~Hz}$. All points on the graph are obtained from the same bilayer sample. There are two main features: The positive and negative branches are asymmetric, and both are approximately linear with $V_{0}$. The error bars show 1 standard deviation corresponding to approximately five measurements of the type of Fig. 9. The solid lines are a fit produced by the model described in the paper.

applied to the same bilayer; one plot (e.g., Fig. 8) contains data all taken from the same bilayer. Because of the inactivation of the ion channels, the recorded peak current is lower than what it would be without inactivation. We correct for the inactivation effect by multiplying with an exponential correction factor that is calculated using the inactivation time of the current trace and the position of the peak. However, since the time scale for inactivation (approximately $400 \mathrm{~ms}$ ) is longer than the rise time of the current corresponding to the stochastic opening of the channels (approximately $50 \mathrm{~ms}$ ), the correction is small.

\section{RESULTS}

We have performed ac experiments according to the protocol of Fig. 6. The number of ion channels in these multichannel recordings is approximately 100 typically, corresponding to currents of order $1 \mathrm{nA}$ at $60-\mathrm{mV}$ polarization. (The conductance of a single KvAP channel at the ionic strength of the experiment is approximately $17 \mathrm{pA} / 100 \mathrm{mV}$.)

The trace of Fig. 9(a) shows the current for a driving signal of $70-\mathrm{mV} \mathrm{rms}$ at $5 \mathrm{~Hz}$. The zero of time in the graph corresponds to when the driving switches from the $-120 \mathrm{mV}$ resting potential to the sinusoidal signal (Fig. 6). At this low frequency, the amplitude of the current decreases to essentially zero within three cycles: This decrease is due to the inactivation process that has a characteristic time scale of approximately $400 \mathrm{~ms}$. Focusing on the first cycle, there is an asymmetry in the positive and negative current responses. At low enough frequencies, the probability that the channel is open, $p(t)$, must follow the 


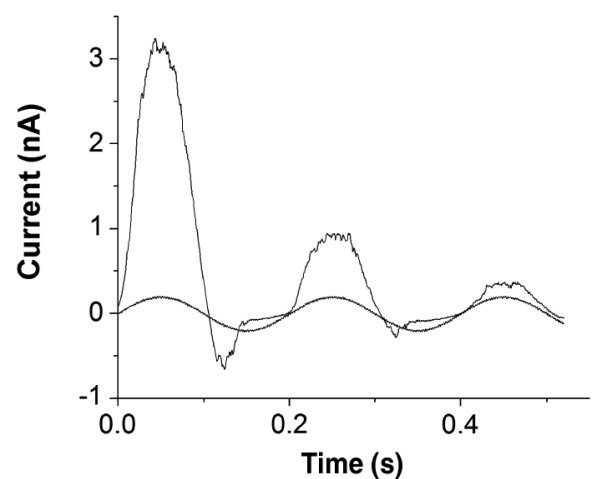

(a)

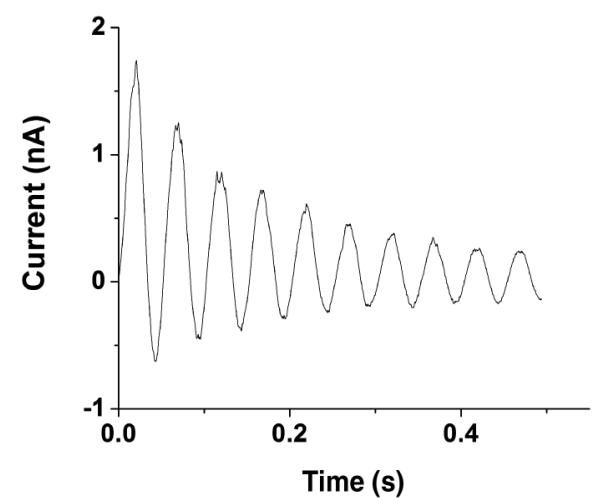

(b)

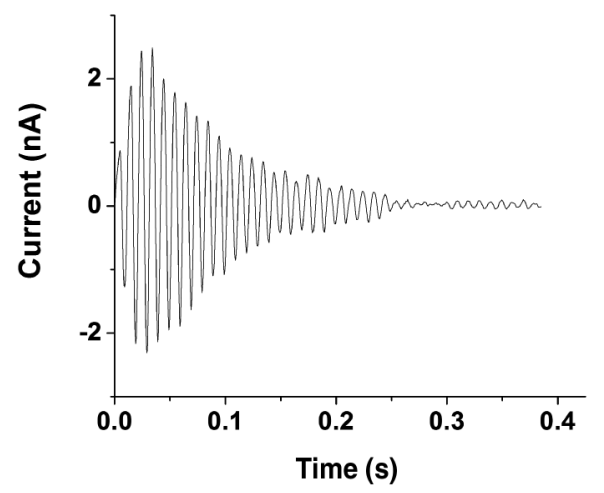

(c)

FIG. 9. Typical current traces from multichannel recordings $(N \sim 100)$ for ac input signals at different frequencies. The three graphs were recorded from different bilayers, with symmetric salt conditions of $150-\mathrm{mM} \mathrm{KCl}$. (a) $5-\mathrm{Hz}$ signal at $70-\mathrm{mV}$ rms amplitude; the applied sinusoidal voltage is plotted on the same graph. (b) $20-\mathrm{Hz}$ signal at $50-\mathrm{mV}$ rms amplitude. At these relatively low frequencies, the positive and negative current swings are asymmetric, reflecting the dc open-probability curve in Fig. 2. (c) $100-\mathrm{Hz}$ signal at $50-\mathrm{mV}$ rms. The envelope of the oscillations first rises, corresponding to the stochastic opening of the channels, then decreases to near zero due to the inactivation process. At these relatively high frequencies, the positive and negative current swings are symmetric.

voltage $V(t)$; thus, the asymmetric waveform is simply a consequence of the dc channel-opening-probability curve in Fig. 2. Figure 9(b) shows the response of the channels to a $20-\mathrm{Hz}$ ac signal at $50-\mathrm{mV}$ rms amplitude. As is evident

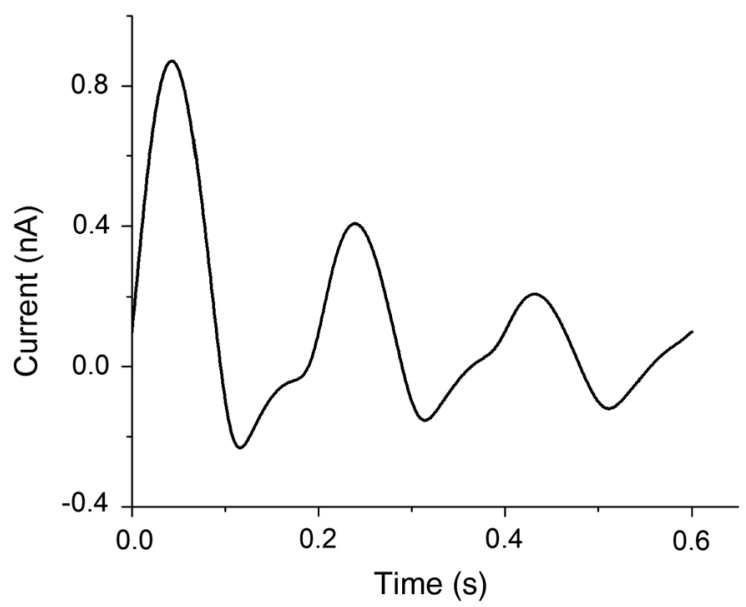

(a)

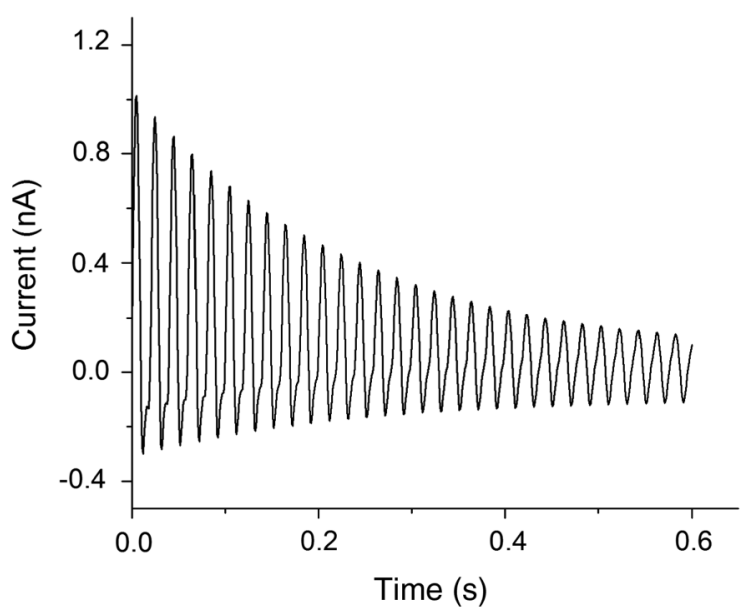

(b)

FIG. 10. Calculated current trace for 100 channels, obtained using the open-probability curve of Fig. 2, Ohm's law, and the exponential decay corresponding to the inactivation process, i.e., $I(t)=N_{\chi} V(t) p[V(t)] \exp (-t / \tau)$, where $N=100$ is the number of channels, $\chi$ is the conductivity of a single channel $(170 \mathrm{pS}), V(t)=V_{0} \sin (2 \pi \nu t)$ is the sinusoidal applied voltage, and $p(V)$ is the open probability at voltage $V$. (a) $\nu=5 \mathrm{~Hz}$; (b) $\nu=50 \mathrm{~Hz}$.

from the graph, the positive and negative of the current swings are still roughly asymmetric.

Figure 9(c) shows the current for a $100-\mathrm{Hz}$ driving signal (of amplitude $50-\mathrm{mV} \mathrm{rms}$ ). The envelope of the oscillation first rises and then decreases. The rise corresponds to the stochastic opening of the channels, similarly to what is observed with dc pulses. The decrease is due to the inactivation process. So, the envelope of the waveform resembles a multichannel current trace for a step depolarizing potential. However, the new feature is that the positive and the negative current swings are now symmetric. If we fold the dc channel-opening probability (Fig. 2) into Ohm's law for the current, we obtain waveforms as shown in Fig. 10. At low frequencies [Fig. 10(a)], the waveform is qualitatively what is seen in the experiments 
[Fig. 9(a)]. But, at high frequencies, simply folding the open-probability curve into Ohm's law [Fig. 10(b)] gives asymmetric positive and negative current swings which are different from what is observed experimentally [Fig. 9(c)].

There are two control parameters in these experiments: the frequency $\nu$ and the amplitude $V_{0}$ of the sinusoidal imposed voltage. We wish to explore the behavior of the system in this parameter space. In order to organize the data, we extract from the traces of Fig. 9 and similar others a single quantity: the peak current. Because of the inherent inactivation of the KvAP ion channel, the peak current we measure is reduced from the value the channel would have without inactivation. We correct for this effect by multiplying the measured peak with an exponential correction factor that is calculated using the measured inactivation time of the current trace and the position of the peak. However, since the time scale for inactivation (approximately $400 \mathrm{~ms}$ ) is longer than the rise time of the current corresponding to the stochastic opening of the channels (approximately $50 \mathrm{~ms}$ ), the correction is small.

In Fig. 8, we show the dependence of the peak current (both for the positive and negative swings) on the amplitude of the applied oscillatory voltage. Figures 8,11 , and 12 show how this graph evolves for increasing frequencies. At $5 \mathrm{~Hz}$ (Fig. 8), the positive and negative peak currents are very asymmetric, but both are essentially linear in the amplitude of the applied voltage $V_{0}$. All the data in this graph were taken from the same bilayer, as are the data of Figs. 11 and 12.

At $40 \mathrm{~Hz}$ (Fig. 11), the two branches (peak positive and negative currents) are still linear with $V_{0}$, but they are now approximately symmetric. At $100 \mathrm{~Hz}$ (Fig. 12), the two branches are still symmetric, but a nonlinear response as a function of the applied voltage $V_{0}$ is evident. The current saturates for $V_{0} \approx 100 \mathrm{mV}$ and even decreases at higher $V_{0}$.

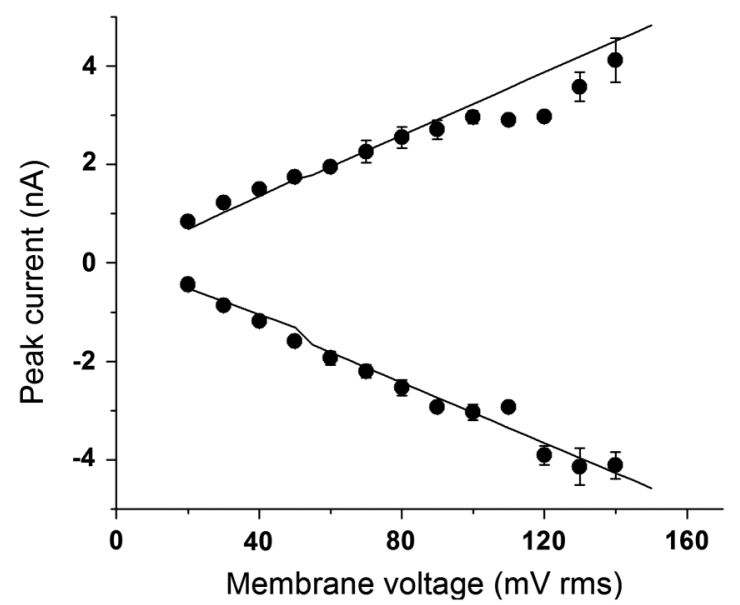

FIG. 11. The peak current measured as in Fig. 8 for $\nu=$ $40 \mathrm{~Hz}$. At this frequency, the two current branches are approximately symmetric and still linear in $V_{0}$. The solid lines represent the model with identical parameter values to those in Fig. 8 except for the excitation frequency $\nu$.

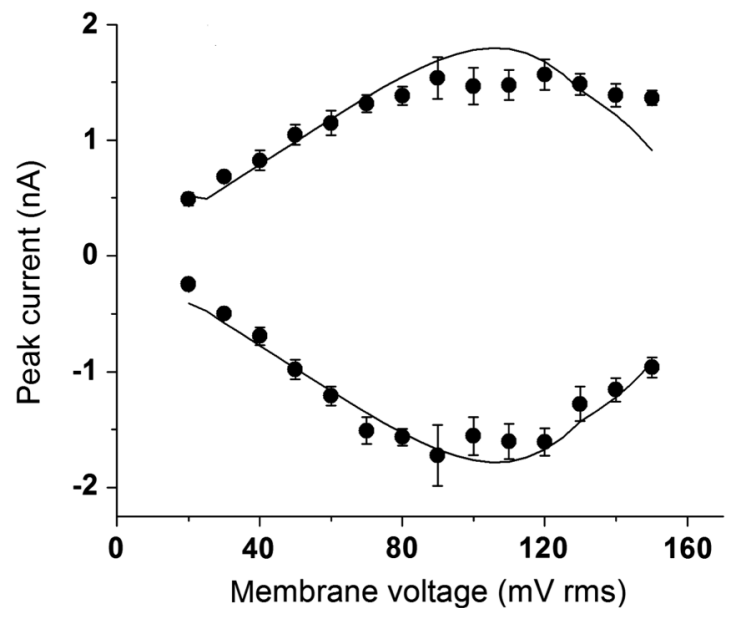

FIG. 12. Peak current for $\nu=100 \mathrm{~Hz}$. The two branches (positive and negative current swings) are approximately symmetric, but the curves are nonlinear in the driving amplitude $V_{0}$. The peak current saturates and even decreases as $V_{0}$ is increased. The solid lines represent the model with identical parameter values to those in Figs. 8 and 11 except for the frequency of excitation $\nu$.

These curves are reversible, so they cannot be accounted for by some disruption of the channels at high voltages.

In Fig. 13, we show the dependence of the peak current (positive swing) on frequency for two different driving voltages. At low applied voltage, the frequency response is essentially flat, while at high applied voltage the peak current decreases with the frequency.

In summary, the experimental results obtained with ac forcing show two striking features. The positive and negative branches of the peak current evolve from asymmetric to symmetric as the frequency is increased. The peak current saturates and even decreases as the amplitude of the ac voltage is increased at high frequencies.

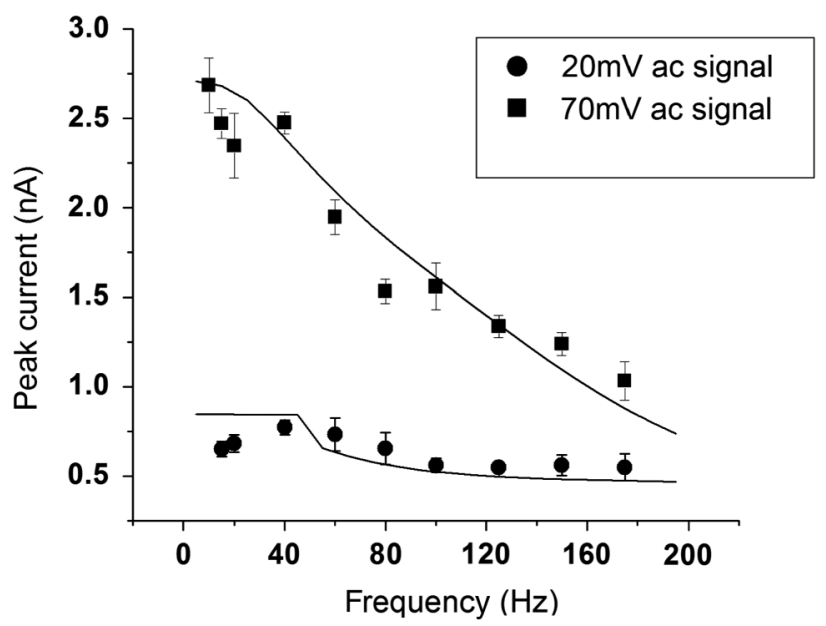

FIG. 13. The dependence of the peak current (positive swing) on the driving frequency for 20 - and $70-\mathrm{mV}$ rms amplitudes of the driving signal. The error bars show 1 standard deviation, and the lines represent the model described in the paper. 
We now introduce a continuum-mechanics model for the operation of the ion channel that allows us to summarize these observations within one simple framework. A similar approach rationalizes the results from our previous nanorheology measurements on the folded state of an enzyme $[17,19]$. Indeed, the initial motivation for the present experiment was to investigate the usefulness of a description in terms of continuum mechanics (i.e., in terms of stiffness and dissipation parameters) applied to the conformational motion of proteins.

As explained in the Introduction, the transmembrane electric field couples to the positively charged arginine residues on the $\mathrm{S} 4$ helix of the voltage-sensing domain of the channel and moves them perpendicular to the membrane. We concentrate on the dynamics of this degree of freedom; i.e., we describe the position of the arginines (supposed to move together) with a coordinate $z$ orthogonal to the bilayer and write an equation of motion for $z$. We assume that $z$ is a continuous variable, whereas the more usual representation of the gating motion is as a two-states system. Both representations are idealizations: The real system has many discrete states. Many discrete states are seen experimentally from measurements of the chargevoltage and conductance-voltage curves [22]. For a twostates system, they should superimpose, but for a real channel they do not. However, a model in terms of several discrete states introduces many parameters that ultimately limit the predictive power. Here, we take the opposite limit to the two-states system, which is infinitely many states.

To write an equation of motion for $z$, we replace all the interactions between the arginines and the rest of the structure with an elastic element and a viscous element in parallel (Fig. 14).

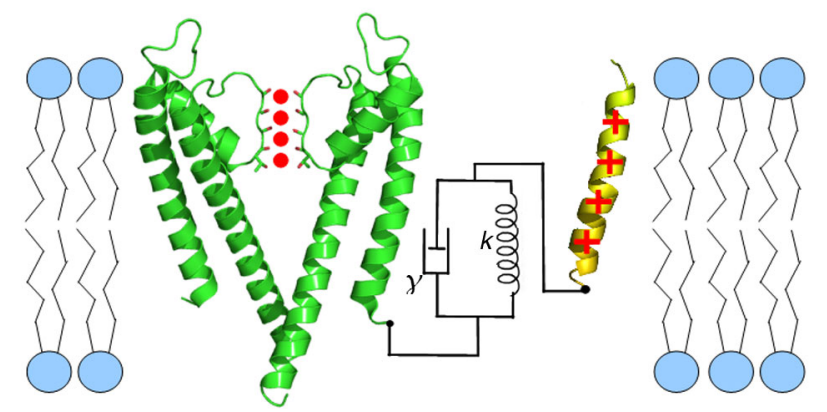

FIG. 14. Conceptual representation of the heuristic mechanical model we use to rationalize the measurements. The positively charged arginines move up and down in response to the electric field generated by the membrane polarization. They are coupled to the rest of the structure through complex interactions that we summarize into one effective mechanical element, a spring, and a dashpot. This mechanical element is nonlinear, with the nonlinearity built into the dissipation coefficient $\gamma$ of the dashpot, which transitions to higher values beyond a critical strain rate $\sigma_{c}$ (see Fig. 16).
The equation of motion for the arginines in one S4 helix (the arginines in one S4 helix are supposed to move together, while the four $\mathrm{S} 4$ helices move simultaneously) is then

$$
\gamma \frac{d z}{d t}+k z=\frac{q V_{0}}{d} \sin (\omega t)
$$

where $k$ is the effective spring constant, $\gamma$ is the dissipation coefficient, and $q$ is the effective number of charges on an S4 helix that move across the bilayer. $d$ is the thickness of the bilayer; $V_{0}$ and $\omega$ are the amplitude and angular frequency of the applied ac voltage across the bilayer. There is no inertial term because inertial effects are negligible at the frequencies of interest. We use a zero-temperature model for simplicity. Adding a thermal-noise term to (1) does not change the average $z$ calculated over many channels and thus has only a small effect on the current $I$. Furthermore, the thermal-noise term is small compared to the driving in the sense that, in the experimental regime,

$$
q V_{0} \approx 4 e \times 50 \mathrm{mV}=200 \mathrm{mV} \gg \mathrm{kT}=25 \mathrm{mV} \text {. }
$$

Also, in this deterministic model, which describes the ensemble-averaged behavior of the channel, the four VSDs of the tetramer move together. The instantaneous current is proportional to the voltage and the number of open channels,

$$
I(t)=N p(t) \chi V(t)
$$

where $N$ is the number of channels, $p(t)$ is the probability that an individual channel is open at time $t, \chi$ is the singlechannel conductance, and $V(t)$ is the membrane potential. We assume that the probability that the channel is open at time $t, p(t)$, is controlled by the instantaneous position of the arginines (i.e., by the variable $z$ ) through the dc channel-opening-probability curve in Fig. 2. In other words, for a dc applied voltage $V$, the displacement of the arginines is (1), $z=q V / k d$, while the channel-opening probability is given by the curve of Fig. 2, which we approximate with a simple sigmoidal function:

$$
p(V)=\frac{1}{2}\left[1+\tanh \left(\frac{V-V_{1}}{\Delta}\right)\right] .
$$

(Fitting the curve of Fig. 2 with this form, which is a FermiDirac distribution, returns the values $V_{1}=-16.1 \mathrm{mV}$ and $\Delta=21.7 \mathrm{mV}$.) Thus, we write $V=(k d / q) z$ in (3) and obtain

$$
p(z)=\frac{1}{2}\left[1+\tanh \left(\frac{(k d / q)\left(z-z_{1}\right)}{\Delta}\right)\right],
$$

where $(k d / q) z_{1}=V_{1}$. The model consists of calculating $z(t)$ from (1) and using it in (4) to obtain $p(t)$ and thus $I(t)$ from (2).

The solution of (1) is

$$
z(t)=\frac{q V_{0}}{\gamma d \sqrt{\omega^{2}+\omega_{0}^{2}}} \sin (\omega t-\phi)+C e^{-\omega_{0} t},
$$


where $\tan (\phi)=\omega / \omega_{0}, \omega_{0}=k / \gamma$, and the constant $C$ is determined by the initial conditions. For $t=0$, the displacement $z$ should correspond to that of the closed channel, $z=z_{0}$ :

$$
C=z_{0}+\frac{q V_{0}}{\gamma d \sqrt{\omega^{2}+\omega_{0}^{2}}} \sin (\phi)
$$

Inserting this solution into (4) and using (2), we find the expression for the current:

$$
I(t)=\frac{N \chi V_{0}}{2}\left\{1+\tanh \frac{\left[(k d / q)\left\{\frac{q V_{0}}{\gamma d \sqrt{\omega^{2}+\omega_{0}^{2}}} \sin (\omega t-\phi)+\left[z_{0}+\frac{q V_{0}}{\gamma d \sqrt{\omega^{2}+\omega_{0}^{2}}} \sin (\phi)\right] e^{-\omega_{0} t}-z_{1}\right\}\right]}{\Delta}\right\} \sin (\omega t) .
$$

The expression for $I(t)$ depends on many parameters; however, in the simulation most are fixed once and for all, as follows: $q=4 \times$ electron charge $=6.4 \times 10^{-19} \mathrm{C}[4]$, $\chi=170 \mathrm{pS}, \quad N=100, d=4 \mathrm{~nm}, \Delta=21.7 \mathrm{mV}$, and $z_{0}=2 \AA$. The parameters that are adjusted to obtain the fits for the graphs in Figs. 8 and 11-13 are $k$ and $\gamma$.

At low enough frequencies, this procedure must yield the experimental response. There is one complication, namely, the transition to the inactive state (Fig. 3), but, if we multiply by the corresponding exponential, we qualitatively obtain the low-frequency response of the channels (Fig. 15).

However, the model (1)-(7) is linear as far as the mechanics of the protein (the $z$ degree of freedom) is concerned: The only nonlinearity is introduced by the response curve (4). Models of this class cannot possibly account for the essential nonlinearity seen in Fig. 12, where as the voltage is increased the current saturates and even decreases. Considering that these curves are reversible (i.e., channels remain intact), it seems that the most plausible mechanism for the saturation and eventual decrease of the current in Fig. 12 is the appearance of a phase lag between the applied voltage and the state of the channel (open or closed) as the voltage is increased. A phase lag that

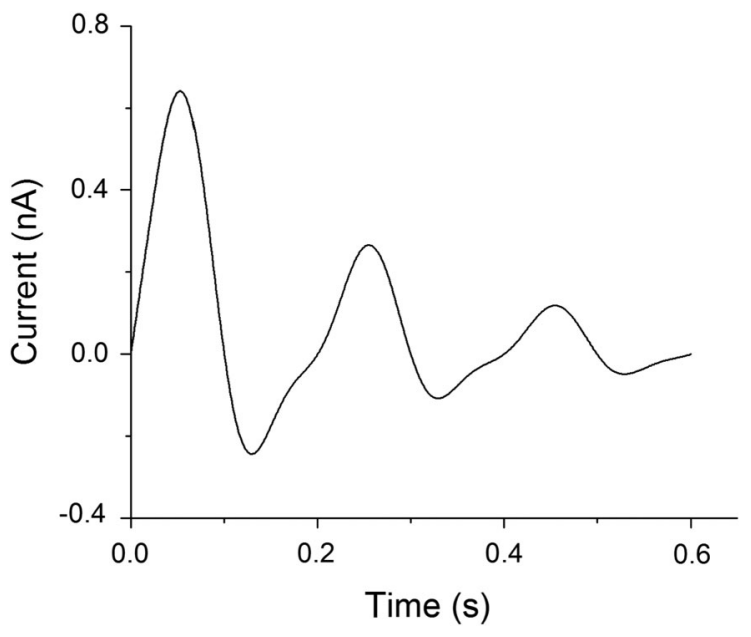

FIG. 15. Low-frequency current trace calculated from the model (1)-(7). The frequency of the driving signal is $\nu=$ $5 \mathrm{~Hz}$. The response is qualitatively similar to the experimental current trace of Fig. 9(a). changes with increasing voltage or frequency is an essential nonlinearity [phase lags for linear equations such as (1) are independent of the amplitude of the forcing] beyond the well-known nonlinearity of the response curve (4).

In our description, we could build this nonlinearity into either the elastic mechanical element (the spring of Fig. 14) or the dissipative mechanical element (the dashpot). The latter choice leads to agreement with all the data. In the end, we find that we can reproduce all of the measurements if we assume a qualitative form of the mechanical nonlinearity, as shown in Fig. 16. The dissipation coefficient $\gamma$ is assumed to be dependent on the shear rate $\sigma=\nu V_{0}$, being constant below a critical shear rate $\sigma_{c}$ and increasing linearly with $\sigma$ for $\sigma>\sigma_{c}$ (Fig. 16), i.e.,

$$
\gamma= \begin{cases}\gamma_{0} & \text { for } \sigma \leq \sigma_{c} \\ \gamma_{0}+\gamma_{1}\left(\sigma-\sigma_{c}\right) / \sigma_{0} & \text { for } \sigma>\sigma_{c} .\end{cases}
$$

Using this function for $\gamma(\sigma)$ in the model (1)-(7), we can now calculate the peak current displayed in Figs. 8 and 11-13 for the various situations. These are the lines in the graphs. In summary, a single set of parameters is used to fit all the data in Figs. 8 and 11-13. These parameters are $\gamma_{0}=0.2 \mathrm{~g} / \mathrm{s}, \quad \gamma_{1}=0.54 \mathrm{~g} / \mathrm{s}, \quad \sigma_{0}=$ $1 \mathrm{Vs}^{-1}, \sigma_{c}=2 \mathrm{Vs}^{-1}, k=20 \mathrm{pN} / \mathrm{nm}, q=6.4 \times 10^{-19} \mathrm{C}$, $\chi=170 \mathrm{pS}, N=100, d=4 \mathrm{~nm}, \Delta=21.7 \mathrm{mV}$, and $z_{0}=2 \AA$.

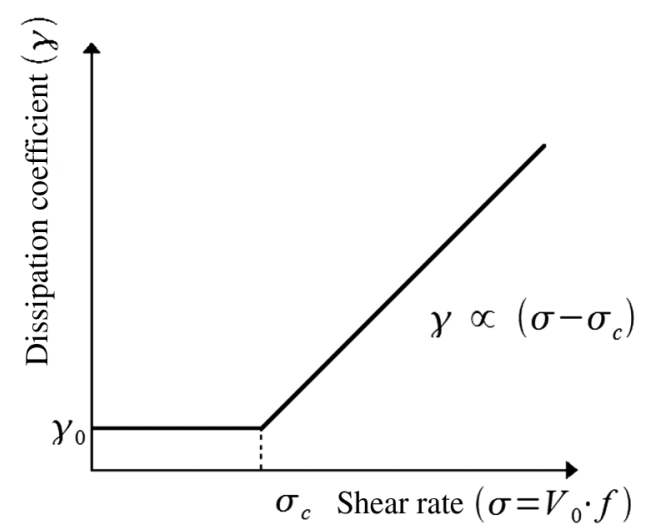

FIG. 16. The nonlinearity heuristically built into the dissipation coefficient $\gamma$. The dissipation coefficient remains constant for shear rates $\sigma$ less than a critical value $\sigma_{c}$. Above the critical value, it increases with a linear dependence on the shear rate. 
With these parameters, the value of $\gamma$ [Eq. (8)] varies from a minimum of approximately $0.2 \mathrm{~g} / \mathrm{s}$ at low frequencies and low forcing amplitudes to a maximum of approximately $6 \mathrm{~g} / \mathrm{s}$ in the opposite limit, within the regime of the experiments.

The behavior of the model is easily understood qualitatively. At low frequencies, the $z$ coordinate is coupled to a simple spring where damping is unimportant, so it follows the voltage swings. These voltage swings are centered around $V=0$, whereas the midpoint of the dc channelopening-probability curve (Fig. 2) is at $V \approx 20 \mathrm{mV}$. Thus, for the positive swings the channels are mostly open and the current is large, while for the negative swings the channels are mostly closed and the current is small. The opening of channels in a positive voltage swing and closing in a negative voltage swing is the origin of the asymmetry in the two branches of the graph of Fig. 8 .

At higher frequencies (Figs. 11 and 12), damping is important (i.e., the $z$ coordinate is coupled to a damped spring), so the amplitude of oscillations in $z$ is small. This small amplitude of oscillation means that the system explores only a small region of the dc channel-openingprobability curve (Fig. 2) around $V=0$. Then, the number of open channels is roughly constant throughout the cycle, so the current is the same for the positive and negative voltage swings; i.e., the two branches in Fig. 11 are symmetric.

At high frequencies (Fig. 12), as $V_{0}$ is increased, eventually $\sigma>\sigma_{c}$ (Fig. 16) and the dissipation coefficient $\gamma$ starts to increase. The increasing dissipation coefficient $\gamma$ introduces a phase lag between the coordinate $z$ and the applied voltage $V$ that increases with $V_{0}$. The phase lag is $\tan (\phi)=\omega / \omega_{0}$, where $\omega_{0}=k / \gamma$, so as $\gamma$ increases $\phi$ moves from close to zero to close to $\pi / 2$. When $\phi=\pi / 2$, when the voltage has a peak the channels are mostly closed and when the channels are mostly open the voltage is going through zero: Thus, the current is always small. This effect accounts for the surprising observation (Fig. 12) that the current saturates and even decreases for increasing $V_{0}$ at high frequencies. Because in a linear system the phase does not depend on the amplitude of the forcing, the only way to obtain this effect is to have a nonlinearity in the system, which here we built, heuristically, on the dissipation coefficient $\gamma$ (Fig. 16).

However, let us now discuss in more detail the extent to which the data of Figs. 8, 11, and 12 determine the parameters of the model. The data of Figs. 8 and 11 do not, by themselves, determine $k$ and $\gamma$ but rather their ratio, i.e., a characteristic angular frequency $\omega_{1}=k / \gamma \approx 150 \mathrm{~s}^{-1}$ (or $\nu_{1}=\omega_{1} / 2 \pi=25 \mathrm{~Hz}$ ) above which dissipation becomes important in the mechanical model. When dissipation becomes important, the amplitude of the arginines' oscillation becomes small and thus the two branches of the peak current become symmetric. The amplitude of the arginine oscillation becomes small between $\nu=5 \mathrm{~Hz}$ and $\nu=40 \mathrm{~Hz}$ (Figs. 8 and 11), so this frequency interval fixes the characteristic frequency $\nu_{1}$ at a value in between. We used $\nu_{1}=25 \mathrm{~Hz}$ in the model. However, within the mechanical model, $k$ is determined by the requirement that a voltage change $\Delta V \approx 50 \mathrm{mV}$ (the width of the openprobability curve in Fig. 2) move the arginines by approximately $10 \AA$; this displacement of $10 \AA$ represents an intermediate value of the different measurements reported for the displacement of the gating charges in the literature, as mentioned in the Introduction. This $10-\AA$ displacement of the gating charge fixes $k \approx 20 \mathrm{pN} / \mathrm{nm}$. Then, $\gamma_{0}=$ $k / \omega_{1} \approx 0.2 \mathrm{~g} / \mathrm{s}$ is also fixed. These parameters are the ones we use in the model to obtain the solid curves in Figs. 8 and 11-13.

On the other hand, the nonlinear behavior of the data in Fig. 12 does not determine the specific form of the nonlinearity [Eq. (8)]. For instance, we find that a $\gamma$ of the form

$$
\gamma= \begin{cases}\gamma_{0} & \text { for } \sigma \leq \sigma_{c} \\ \gamma_{0}+\gamma_{1}\left[\left(\sigma-\sigma_{c}\right) / \sigma_{0}\right]^{n} & \text { for } \sigma>\sigma_{c}\end{cases}
$$

can also fit the data of Figs. 8 and 11-13 equally well, with $n$ ranging from 0.5 to 2 and correspondingly opportune values for $\gamma_{1}$ and $\sigma_{c}$. The main requirement to fit the data is that $\gamma$ be constant below a critical shear rate $\sigma_{c}$ and increase sufficiently fast above $\sigma_{c}$.

\section{DISCUSSION}

This paper contains two main points. The first is that it is interesting to investigate the response of a voltage-gated channel to an applied oscillating voltage. The response to a voltage step does not tell the whole story because the system is nonlinear.

The second result is that we discover a fundamental nonlinearity in the mechanics of the channel. By "fundamental," we mean the following. There is of course a wellknown "nonlinear" effect in the operation of the channel, which is manifested in the dc channel-opening-probability curve in Fig. 2. However, that response is characteristic of any two-states system in statistical mechanics: Where the energy difference between the two states is a linear function of the control parameter (here the applied voltage), the result is a Fermi-Dirac distribution for the probability to be in one particular state as a function of the control parameter; this Fermi-Dirac probability distribution is essentially Fig. 2. In contrast, the nonlinear response shown in Fig. 12 arises because the properties of the system (here the mechanical parameters) change with increasing amplitude of the forcing.

The essence of this nonlinearity is suggested by the data of Fig. 12: There must be a phase lag between the opening of the channels and the applied voltage, and this phase lag must increase with increasing the forcing amplitude. It is hard to imagine an alternative scenario by which one can obtain a current that decreases as the voltage is increased. 
The specific model we propose to describe the data is of course heuristic; more complicated mechanical models are possible, but here we just present the simplest we have found that describes the data. Within this model, we describe the position of the charges (the arginines on the S4 helix) that are known to move during gating with the continuous variable $z$ and write an effective equation of motion for $z$ by coupling it to a mechanical element (a spring and dashpot in parallel). In short, we introduce two effective parameters, a spring constant $k$ and a dissipation coefficient $\gamma$, but $\gamma$ depends on the shear rate, which introduces an essential nonlinearity in the system. The other parameters that enter the model (the gating charge, the thickness of the bilayer, etc.) are essentially known experimentally, and we use those values. Then, all experimental data of this study are fit by the same values for $k$ and $\gamma$. We fix $k=20 \mathrm{pN} / \mathrm{nm}$, corresponding to a de displacement of the arginines of $10 \AA$ at $50 \mathrm{mV}$. Then, we find $\gamma_{0} \sim$ $0.2 \mathrm{~g} / \mathrm{s}$. The magnitude of the oscillation of the charges in the simulation is in the range of approximately 10-25 $\AA$. There are previous experiments where the displacement of the arginines was measured using Förster-resonanceenergy-transfer and luminescence-resonance-energytransfer techniques [8,9]; these experiments show displacements of the arginines of approximately $2 \AA$, whereas voltage-clamp fluorometry studies [10] obtained displacements of approximately $6-8 \AA$ and biotin-avidin accessibility studies indicated larger movement of approximately 15-20 $\AA$ [11]. We use an average displacement of $10 \AA$ considering these different experimental measurements and use this value to find the spring constant $k$ in the model. This value of $k$ determines the magnitude of the oscillation of the gating charges in the simulation.

This model for the gating charge displacement is a zerotemperature model, and one might wonder why it reproduces the average behavior of an ensemble of channels so well. The reason is that, from the point of view of the mechanics, adding a thermal-noise term to Eq. (1) (i.e., writing a Langevin equation instead of a deterministic equation) hardly modifies the ensemble-averaged response (see, e.g., the Appendix in [19]), while, from the point of view of the coupling to the channel-opening-probability curve (Fig. 2), thermal motion is not very important compared to the driving force in the regime of the experiments $\left(V_{0} \sim 50 \mathrm{mV}\right)$ because $50 \mathrm{mV} \times 4 e=200 \mathrm{meV} \gg$ $\mathrm{kT}=25 \mathrm{meV}$.

Our interpretation of the results assumes that the active ion channels in the bilayer are orientated in the same direction; these oriented channels are depolarized when we step the voltage to $+60 \mathrm{mV}$ from $-120 \mathrm{mV}$. Actually, even if the channels were not oriented in the bilayer, the measurements would look the same because the recovery rate from inactivation for the KvAP ion channel is slow [4] compared to the time scales of our voltage protocol. Ion channels in the opposite orientation will get inactivated

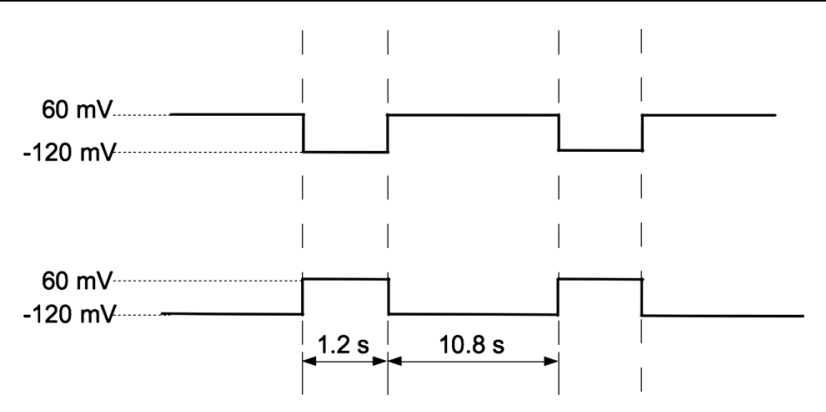

FIG. 17. The voltage protocols to determine the proportion of channels in the two orientations. The holding voltage of $60 \mathrm{mV}$ in the top pulse inactivates all channels in the correct orientation. Thus, a step of $-120 \mathrm{mV}$ opens all the oppositely oriented channels while the channels oriented in the other direction are inactivated. The bottom pulse does the reverse and opens only the channels in the correct orientation.

during the $10.8 \mathrm{~s}$ holding step at $-120 \mathrm{mV}$. These channels need longer than $1.2 \mathrm{~s}$ (the duration of the ac pulse) of a hyperpolarizing voltage step to recover from inactivation. (The recovery time is approximately tens of seconds [4].) Therefore, the contribution from the oppositely oriented channels to the ac current trace is negligible. But, regardless, the channels in our setup are in fact oriented, with the population of channels in the opposite orientation being only about $8 \%$. This value was determined by applying the voltage protocol shown in Fig. 17 to obtain the percentage of opposite channels in one bilayer and then averaging the value over five different bilayers. This voltage protocol (Fig. 17) can be used to find the proportion of channels in the two orientations, as the top pulse protocol fully inactivates the channels in the right orientation and completely opens the channels in the opposite orientation, while the bottom pulse protocol fully inactivates the channels in the opposite orientation and completely opens the channels in the right orientation.

The model we propose has one characteristic time scale $\tau=2 \pi \gamma / k \approx 40 \mathrm{~ms}$. The corresponding time scale in the physical system is the rise time of the multichannel current corresponding to the stochastic opening of the channels after a depolarizing voltage step. At frequencies below $1 / \tau$, the channels simply "follow" the ac drive according to the dc channel-opening-probability curve of Fig. 2. The new results of the paper concern the behavior at frequencies above $1 / \tau$. In previous models [23], based on the energy landscape description, the same physical time scale $\tau$ is constructed from the rate of crossing a barrier, essentially $1 / \tau \propto(1 / \eta) \exp (-E / T)$, where $E$ is the energy barrier and $\eta$ the internal viscosity. Because of the presence of the Arrhenius factor, the effective viscosity $\eta$ in this description is much smaller than the effective viscosity in our description, where there is no barrier. For example, Grabe et al. [23] calculate an approximate barrier height for the motion of the S4 helix by computing electrostatic energies. Then, they use the Kramers reaction rate theory 
to arrive at the time scale $\tau$, thereby obtaining a much larger value for the effective diffusion coefficient of the S4 helix than we would infer from our description. Which of these two alternative approaches is more useful in describing the dynamics of this system is one question we wish to pose with this study.

An alternative interpretation of the heuristic model (1)-(7) is to consider that for an individual channel the position of the arginines is a two-states (or "few-states") variable, while the continuous coordinate $z$ in (1) represents the average position over many channels. Then, the question is why it is that this averaged variable $z$ seems to at least approximately obey Eq. (1). Perhaps this agreement of the averaged value of $z$ with Eq. (1) can be justified through a calculation in which two potential wells representing two states of the VSD are oscillated up and down by the applied voltage. However, in this paper, we do not supply a microscopic justification of the heuristic model (1)-(7), and in any case a two-states model is as much of an idealization as a continuum model, so this question is left open.

In the nanorheology experiments described in $[17,19]$, we apply an oscillating mechanical force to an enzyme (Guanylate Kinase) in the native state and measure the resulting amplitude of the deformation. The present experiment is conceptually similar and can also be viewed as a "nanorheology" measurement. Namely, we drive the motion of the S4 helix (or parts of the voltage-sensing domain) with an oscillating force (generated by the electric field across the membrane) and measure the response. In both cases, we can quantitatively summarize the results in terms of continuum-mechanics models, which means introducing an effective stiffness parameter $k$ and an effective dissipation parameter $\gamma$. These parameters are effective parameters that describe the ensemble-averaged trajectories of the system. The underlying microscopic physical processes must involve local interaction potentials between nearby residues and the effectively dissipative process of hopping from one local minimum to the next as the conformation changes. The mechanical similarity between the ion channel and the enzyme is that in both cases the ensemble-averaged mechanics contains both elasticity and dissipation. Also, the values of these parameters $(k$ and $\gamma)$ are similar in the two cases, reflecting perhaps a universal scale of the microscopic local energy minima and barriers between them or, in short, the fact that the same interactions hold together the two structures. However, there are also differences: In one case, the ensemble-averaged behavior is described by a series combination of elastic and dissipative elements; in the other, it is described by a parallel combination. We offer the following reflection: Any mechanical system composed of many coupled springs and dashpots will behave as either one effective spring and dashpot in series or as a spring and dashpot in parallel.
While the details of the mechanical model employed to summarize the measurements are different for the enzyme and the channel, one important physical effect is qualitatively the same: There is a range of forces where the system becomes more viscous, or more solidlike, at high frequencies. For the channel, we qualitatively represent this range of forces for which the system becomes more viscous or solidlike in Fig. 16, where the dissipation coefficient we use in the model increases with shear rate. While such "shear thickening" is well known in certain colloidal suspensions and polymeric materials [24], where it can lead to rather spectacular effects, the observation of shear thickening in a molecule is unexpected. The microscopic mechanism of shear thickening generally involves shearinduced clustering of the particles that compose the system or, one could say, a transient rearrangement of the bonds between different parts of the system (see, e.g., [25]). Whether a similar mechanism is responsible for the behavior of the ion channel reported here is an interesting open question.

It is also noteworthy that in both cases (the channel and the enzyme) the experimental data actually fix the ratio $k / \gamma$ and that this characteristic frequency comes out the same $[\nu=(1 / 2 \pi) k / \gamma \approx 20 \mathrm{~Hz}]$ for these two very different systems and measurements. In [17], we assumed a value of $k=5 \mathrm{pN} / \mathrm{nm}$ and therefore obtained $\gamma \approx$ $5 \times 10^{-2} \mathrm{~g} / \mathrm{s}$. Here, we assume $k=20 \mathrm{pN} / \mathrm{nm}$ and therefore obtain $\gamma \approx 0.2 \mathrm{~g} / \mathrm{s}$. Thus, the value of the internal dissipation coefficient $\gamma$ is, as an order of magnitude, remarkably consistent between these two very different proteins and measurement methods. On the other hand, measurements of the internal dissipation from the refolding rates of fast-folding proteins [26,27] give values seemingly 8 orders of magnitude smaller. We proposed in [17] that this very small internal dissipation is due to the viscoelastic nature of the protein's mechanics.

In conclusion, we find experimentally a fundamental nonlinearity in the mechanics of the KvAP voltage-gated ion channel, which is summarized macroscopically by the model (1)-(7).

\section{ACKNOWLEDGMENTS}

We thank Professor MacKinnon for the gift of the KvAP plasmid. We also thank the anonymous referees for their thoughtful comments. This work was supported by the UC Lab Research Program, by NSF Grant No. DMR-1006162, and by the U.S.-Israel Binational Science Foundation under Grant No. 2010448.

[1] Franklin Bretschneider and Jan R. De Weille, Introduction to Electrophysiological Methods and Instrumentation (Academic, New York, 2006).

[2] Eric N. Ervin, Ryuji Kawano, Ryan J. White, and Henry S. White, Simultaneous Alternating and Direct Current 
Readout of Protein Ion Channel Blocking Events Using Glass Nanopore Membranes, Anal. Chem. 80, 2069 (2008).

[3] M. Misakian, J.J. Kasianowicz, B. Robertson, and O. Petersons, Frequency Response of Alternating Currents through the Staphylococus aureus $\alpha$-hemolysin Ion Channel, Bioelectromagnetics (N.Y.) 22, 487 (2001).

[4] Vanessa Ruta, Youxing Jiang, Alice Lee, Jiayun Chen, and Roderick MacKinnon, Functional Analysis of an Archaebacterial Voltage-Dependent $\mathrm{K}^{+}$Channel, Nature (London) 422, 180 (2003).

[5] Youxing Jiang, Alice Lee, Jiayun Chen, Vanessa Ruta, Martine Cadene, Brian T. Chait, and Roderick MacKinnon, X-Ray Structure of a Voltage-Dependent $K^{+}$Channel, Nature (London) 423, 33 (2003).

[6] Youxing Jiang, Vanessa Ruta, Jiayun Chen, Alice Lee, and Roderick MacKinnon, The Principle of Gating Charge Movement in a Voltage-Dependent $\mathrm{K}^{+}$Channel, Nature (London) 423, 42 (2003).

[7] Francisco Bezanilla, Voltage-Gated Ion Channels, IEEE Trans. Nanobiosci. 4, 34 (2005).

[8] Baron Chanda, Osei Kwame Asamoah, Rikard Blunck, Benoit Roux, and Francisco Bezanilla, Gating Charge Displacement in Voltage-Gated Ion Channels Involves Limited Transmembrane Movement, Nature (London) 436, 852 (2005).

[9] David J. Posson, Pinghua Ge, Christopher Miller, Francisco Bezanilla, and Paul R. Selvin, Small Vertical Movement of a $\mathrm{K}^{+}$Channel Voltage Sensor Measured with Luminescence Energy Transfer, Nature (London) 436, 848 (2005).

[10] M. M. Pathak, V. Yarov-Yarovoy, G. Agarwal, B. Roux, P. Barth, S. Kohout, F. Tombola, and E. Y. Isacoff, Closing in on the Resting State of the Shaker $K(+)$ Channel, Neuron 56, 124 (2007).

[11] V. Ruta, J. Chen, and R. Mackinnon, Calibrated Measurement of Gating-Charge Arginine Displacement in the KvAP Voltage-Dependent $K(+)$ Channel, Cell 123, 463 (2005).

[12] L. Kiss and S. J. Korn, Modulation of C-Type Inactivation by $K^{+}$at the Potassium Channel Selectivity Filter, Biophys. J. 74, 1840 (1998).

[13] Francisco Bezanilla, The Voltage Sensor in VoltageDependent Ion Channels, Physiol. Rev. 80, 555 (2000).

[14] Francesco Tombola, Medha M. Pathak, and Ehud Y. Isacoff, How Does Voltage Open an Ion Channel?, Annu. Rev. Cell Dev. Biol. 22, 23 (2006).
[15] N. E. Schoppa, K. McCormack, M. A. Tanouye, and F. J. Sigworth, The Size of Gating Charge in Wild-Type and Mutant Shaker Potassium Channels, Science 255, 1712 (1992).

[16] B. L. Tempel, D. M. Papazian, T. L. Schwarz, Y. N. Jan, and L. Y. Jan, Sequence of a Probable Potassium Channel Component Encoded at Shaker Locus of Drosophila, Science 237, 770 (1987).

[17] Yong Wang and Giovanni Zocchi, The Folded Protein as a Viscoelastic Solid, Europhys. Lett. 96, 18003 (2011).

[18] Yong Wang and Giovanni Zocchi, Elasticity of Globular Proteins Measured from ac Susceptibility, Phys. Rev. Lett. 105, 238104 (2010).

[19] Yong Wang and Giovanni Zocchi, Viscoelastic Transition and Yield Strain of the Folded Protein, PLoS ONE 6, e28097 (2011).

[20] L. Heginbotham, M. LeMasurier, L. KolmakovaPartensky, and C. Miller, Single Streptomyces lividans $k^{+}$Channels, J. Gen. Physiol. 114, 551 (1999).

[21] W. Wonderlin, A. Finkel, and R. French, Optimizing Planar Lipid Bilayer Single-Channel Recordings for High Resolution with Rapid Voltage Steps, Biophys. J. 58, 289 (1990).

[22] Sandipan Chowdhury and Baron Chanda, Estimating the Voltage-Dependent Free Energy Change of Ion Channels Using the Median Voltage for Activation, J. Gen. Physiol. 139, 3 (2011).

[23] M. Grabe, H. Lecar, Y.N. Jan, and L. Y. Jan, A Quantitative Assessment of Models for VoltageDependent Gating of Ion Channels, Proc. Natl. Acad. Sci. U.S.A. 101, 17640 (2004).

[24] Norman J. Wagner and John F. Brady, Shear Thickening in Colloidal Dispersions, Phys. Today, 62, No. 10, 27 (2009).

[25] Donghua Xu, Jennifer L. Hawk, David M. Loveless, Sung Lan Jeon, and Stephen L. Craig, Mechanism of Shear Thickening in Reversibly Cross-Linked Supramolecular Polymer Networks, Macromolecules 43, 3556 (2010).

[26] Troy Cellmer, Eric R. Henry, James Hofrichter, and William A. Eaton, Measuring Internal Friction of an Ultrafast-Folding Protein, Proc. Natl. Acad. Sci. U.S.A. 105, 18320 (2008).

[27] K. W. Plaxco and D. Baker, Limited Internal Friction in the Rate-Limiting Step of a Two State Protein Folding Reaction, Proc. Natl. Acad. Sci. U.S.A. 95, 13591 (1998). 